\title{
Formação em serviço: a atuação do enfermeiro em um Programa de Residência Multiprofissional em Saúde no Oeste do Pará
}

\author{
In-service training: the role of nurses in a multiprofessional residency program in health in the \\ west of Pará \\ Formación en servicio: el papel de los enfermeros en un programa de residencia multiprofesional \\ en salud en el oeste de Pará
}

\section{Resumo}

Objetivo: relatar a experiência de uma residente de enfermagem no Programa de Residência Multiprofissional (RMS) em Atenção Integral em Ortopedia e Traumatologia. Metodologia: Trata-se de um relato de experiência desenvolvido a partir das experiências vivenciadas no período de março de 2020 até janeiro de 2022, em dois hospitais públicos no município de Santarém, região Oeste do Pará. A análise deste estudo ocorreu de forma crítica e reflexiva e por não se tratar de pesquisa, não foi submetido ao Comitê de Ética em Pesquisa. Resultados: A atuação do residente de enfermagem é realizada através de rodízios nos variados setores das instituições hospitalares como, centro cirúrgico, clínica médica e cirúrgica, setor de urgência e emergência no trauma e ambulatório de ortopedia, com pacientes pediátricos, adultos e idosos. Envolve a avaliação de enfermagem, cuidados pré, trans e pós-operatório, cuidado ao paciente politraumatizado, confecção de imobilizações ortopédicas, instrumentação cirúrgica, curativo e orientações para o autocuidado. Considerações Finais: conclui-se que a RMS contribui para aquisição de conhecimentos técnicoscientíficos que possibilitam uma nova conduta diante da prática profissional, tornando o residente apto a prestar uma 
assistência segura e sistematizada no serviço de saúde e atenção aos usuários com afecções ortotraumatológicas no âmbito do Sistema Único de Saúde.

Palavras-chave: Enfermagem; Formação em saúde; Residência multiprofissional; Ortopedia; Traumatologia.

\begin{abstract}
Objective: to report the experience of a nursing resident in the Multiprofessional Residency Program (RMS) in Comprehensive Care in Orthopedics and Traumatology. Methodology: This is an experience report developed from experiences from March 2020 to January 2022, in two public hospitals in the city of Santarém, western region of Pará. The analysis of this study took place in a critical and reflective way and as it was not a research, it was not submitted to the Research Ethics Committee. Results: The role of the nursing resident is carried out through rotation in the various sectors of hospital institutions such as the operating room, medical and surgical clinic, urgency and emergency sector in trauma and orthopedics outpatient clinic, with pediatric, adult and elderly patients. It involves nursing assessment, pre, trans and postoperative, care for multiple trauma patients, making orthopedic immobilizations, surgical instrumentation, dressing and guidelines for self-care. Final Considerations: It is concluded that the RMS contributes to the acquisition of technical-scientific knowledge that allows a new conduct in the face of professional practice, making the resident able to provide safe and systematized assistance in the health service and attention to users with orthotraumatological disorders within the scope of the national health service.
\end{abstract}

Keywords: Nursing; Health training; Multiprofessional residency; Orthopedics; Traumatology.

\begin{abstract}
Resumen
Objetivo: relatar la experiencia de una residente de enfermería en el Programa de Residencia Multiprofesional (RMS) en Atención Integral en Ortopedia y Traumatología. Metodología: Este es un relato de experiencia desarrollado a partir de experiencias de marzo de 2020 a enero de 2022, en dos hospitales públicos de la ciudad de Santarém, región occidental de Pará.El análisis de este estudio se realizó de forma crítica y reflexiva y como no fue una investigación, no fue sometido al Comité de Ética en Investigación. Resultados: El papel del residente de enfermería se realiza a través de la rotación en los diversos sectores de las instituciones hospitalarias como el quirófano, la clínica médica y quirúrgica, el sector de urgencias y emergencias en el ambulatorio de trauma y ortopedia, con pacientes pediátricos, adultos y adultos mayores. Implica valoración de enfermería, cuidados pre, trans y postoperatorios, atención al politraumatizado, realización de inmovilizaciones ortopédicas, instrumentación quirúrgica, vendajes y orientaciones para el autocuidado. Consideraciones finales: Se concluye que la RMS contribuye a la adquisición de conocimientos técnico-científicos que permitan una nueva conducta frente al ejercicio profesional, capacitando al residente para brindar asistencia segura y sistematizada en el servicio de salud y atención a los usuarios con trastornos ortotraumatológicos dentro del alcance del Sistema Único de Salud.
\end{abstract}

Palabras clave: Enfermería; Formación en salud; Residencia multiprofesional; Ortopedía; Traumatología.

\title{
1. Introdução
}

A residência multiprofissional em saúde (RMS) é uma modalidade de especialização lato sensu instituída a partir da promulgação da Lei ${ }^{\circ} 11.129$ de 2005 que tem como principal característica a formação em serviço, com 60 horas de carga horária semanal destinadas à atividades práticas e teórico-práticas e duração de 24 meses (Brasil, 2006). Nesse sentido, a possibilidade de formação em serviço busca a produção do conhecimento através da vivencia diária dos profissionais para aperfeiçoar a qualidade do atendimento ao usuário do Sistema Único de Saúde (SUS) (Arnemann et al., 2021).

À vista disso, a RMS tem como objetivo promover a formação de profissionais da saúde, com exceção da médica, a partir da prática profissional, de maneira a tornar o residente preparado para questionar e construir novos modelos de assistência e exercício profissional com qualidade nas áreas de cuidado integral à saúde. Assim, busca-se a excelência e integralidade do serviço prestado, de modo a possibilitar mudanças nas relações, nos processos e nas respostas às necessidades de saúde da população (Torres et al., 2019; Silva \& Natal, 2019).

Os programas de residências oportunizam a problematização da realidade no cotidiano dos serviços de saúde e articulação com as instituições de ensino para a integração de residentes, docentes, gestores, profissionais da saúde e usuários do SUS (Arnemann et al., 2018). Dessa forma, o ambiente hospitalar constitui-se como um importante campo de assistência/cuidado para a formação de residentes (Seltenreich, 2017).

No município de Santarém, região Oeste do Pará, foi implantado no ano de 2013 o Programa de Residência Multiprofissional em Saúde na área de Atenção Integral em Ortopedia e Traumatologia que é vinculado a Universidade do 
Estado do Pará (UEPA) e Hospital Regional do Baixo Amazonas (HRBA) e tem como principal objetivo a formação de especialistas capacitados para promover ações integradas no atendimento ambulatorial, hospitalar, como também no âmbito da saúde do politraumatizado, com visão holística do paciente com afecções ortotraumatológicas. O programa atualmente inclui vagas para terapeutas ocupacionais, psicólogos, assistentes sociais, farmacêuticos, fisioterapeutas e enfermeiros (Galvão, 2017; Barbosa et al., 2021).

Nesse cenário, o trauma ortopédico é um problema de saúde pública que atinge milhares de pessoas todos os anos no mundo, ocasionando condições mórbidas, internações hospitalares e altos custos com tratamentos, comprometendo a função do indivíduo e sua participação econômica na sociedade (Santos et al., 2021). Assim, os acometimentos ortopédicos e traumatológicos representam uma das principais demandas de pacientes atendidos na região Oeste do Pará que dispõe da parceria da RMS no serviço e na atenção à saúde para a assistência de usuários no SUS.

Desse modo, a atuação do enfermeiro no cuidado ao paciente com afecções ortopédicas e traumatológicas é um campo especializado, regulamentado pela Resolução do Conselho Federal de Enfermagem no 422 de 2012 e corresponde à assistência em situações de doenças, processos congênitos e do desenvolvimento, traumas, infecções e outros comprometimentos que atingem o sistema musculoesquelético, articular e o tecido conjuntivo de suporte. Problemas de saúde clínicos, de reabilitação e cirúrgicos, que devem ser assistidos por profissionais de Enfermagem devidamente capacitados (Robleda et al., 2014, Silva, 2017).

Portanto, a formação de profissionais especializados se torna imprescindível diante deste panorama e sinaliza-se a importância de expressar as ações e intervenções do enfermeiro atuante na Residência Multiprofissional em Saúde, com destaque para a área de ortopedia e traumatologia. Considerando a importância deste processo, o estudo objetivou relatar a experiência de uma residente de enfermagem no Programa de Residência Multiprofissional em Atenção Integral em Ortopedia e Traumatologia no Oeste do Pará e propõe-se a descrever e ampliar o entendimento sobre a formação em serviço do enfermeiro residente a partir de experiência vivenciada no serviço e na atenção à saúde.

\section{Metodologia}

Trata-se de um estudo de caráter descritivo e observacional do tipo relato de experiência desenvolvido a partir das vivencias de uma residente de enfermagem na Residência Multiprofissional em Atenção Integral em Ortopedia e Traumatologia vinculada a Universidade do Estado do Pará (UEPA), no período de março de 2020 até janeiro de 2022, no município de Santarém, região Oeste do Estado do Pará, em diversos setores de assistência ao paciente traumato-ortopédico em dois hospitais públicos, Hospital Municipal de Santarém Dr. Alberto Tolentino Sotelo (HMS) e Hospital Regional do Baixo Amazonas Dr. Waldemar Penna (HRBA).

O relato de experiência é um tipo de produção de conhecimento que aborda uma vivência acadêmica e/ou profissional que tem como característica a descrição de intervenções com reflexão crítica e embasamento científico. As experiências podem ser de pesquisas, estágios, ensino, projetos de extensão universitária, assim como também no área da pós-graduação, como na Residência Multiprofissional em Saúde (Ossege et al., 2020, Mussi et al., 2021).

O HRBA trata-se de um hospital Estadual de média e alta complexidade classificado como ONA 3, acreditado com excelência, concebido pela Organização Nacional de Acreditação, que conta com setores de Clínica Pediátrica, Obstétrica, Cirúrgica, Oncológica e Médica; Unidade de Terapia Intensiva (UTI) neonatal, pediátrica e adultos; Centro Cirúrgico; hemodiálise; hemodinâmica; quimioterapia; radioterapia; radiodiagnóstico por imagem; pediatria; métodos gráficos; laboratório de Análises Clínicas e Ambulatório de Especialidades.

Já o HMS, é uma instituição municipal que assiste pacientes de baixa e média complexidade e possui setores de Clínica Pediátrica, Obstétrica, Cirúrgica e Médica, Urgência e Emergência; Unidade de Terapia Intensiva (UTI) adulto, 
pediátrica e adultos; Centro Cirúrgico; Setor de Ortopedia e Traumatologia (SOT); Farmácia Hospitalar; Hemodiálise; Radiodiagnóstico por imagem; Laboratório de Análises Clínicas e Ambulatório de Especialidades. Devido ao seu perfil, o hospital atende uma demanda muito grande de cirurgias de urgência, notadamente na área do trauma e das patologias cirúrgicas agudas.

Ambos são hospitais públicos de atendimento exclusivo ao SUS, considerados hospitais de ensino utilizados como campo de estágio pelas instituições de ensino superior. Atendem usuários do município de Santarém e de toda a região de abrangência da $9^{\text {a }}$ Regional da Saúde, através do sistema de regulação de diversas especialidades, provenientes do Oeste do Estado do Pará, sendo 20 cidades que compõem essa região que se divide em Baixo Amazonas (12 cidades) e Região do Tapajós (8 cidades) e assistem usuários com diversas afecções ortotraumatológicas para tratamento ambulatorial, clínico e cirúrgico.

Assim, a atuação de Enfermagem na RMS em ortopedia e traumatologia no Oeste do Pará é realizada através de rodízios nos variados setores dessas instituições hospitalares como, centro cirúrgico, clínica médica e cirúrgica, setor de urgência e emergência no trauma e ambulatório de ortopedia, com pacientes pediátricos, adultos e idosos.

A análise deste estudo ocorreu de forma crítica e reflexiva, e por não se tratar de pesquisa, não foi submetido ao Comitê de Ética em Pesquisa (CEP).

\section{Resultados e Discussões}

\subsection{O Programa de Residência Multiprofissional em Saúde de Ortopedia e Traumatologia no Oeste do Pará}

Para o ingresso na Residência Multiprofissional em Saúde é necessário a aprovação em processo seletivo pela Universidade do Estado do Pará, com prova teórica e análise curricular que anualmente são ofertadas 04 vagas para a categoria de Enfermagem em Ortopedia e Traumatologia. Após a aprovação e tramites para a matrícula, o residente é recebido no HRBA para uma semana de integração, onde são apresentados normas, fluxo da instituição, deveres e funcionamento da residência. $\mathrm{O}$ programa possui carga horária de 60 horas semanais e 12 horas diárias, distribuídas de segunda a sexta-feira, perfazendo o total de 5.640 horas que são divididas em dois anos, ocorrendo $80 \%$ da carga horária prática para a formação em serviço e $20 \%$ destinadas para atividades teórico-práticas.

O residente é contemplado por uma bolsa de estudo mensal disponibilizada pelo Ministério da Educação, sendo que, devido a pandemia da Covid-19, também foi beneficiado com uma bonificação referente ao Programa Brasil Conta Comigo Residentes que teve duração de abril de 2020 até o mês de dezembro de 2021. Além disso, a partir de dezembro de 2021, o governo do Pará, como incentivo para os profissionais da saúde em formação principalmente no contexto atual, instituiu o Programa Estadual "Qualifica Saúde”, por meio da Secretaria de Estado de Saúde Pública (SESPA) e UEPA.

A RMS de Enfermagem no Oeste do Pará conta com preceptores vinculados as instituições hospitalares, tutora, coordenação da residência local em Santarém e está associada a Coordenação geral da Residência Multiprofissional (COREMU) localizada em Belém do Pará. Porém, observa-se que há a ausência de atividades envolvendo todos os preceptores, tutores e residentes, sendo uma limitação do programa, visto que Dias et al. (2016) destacam que é muito importante essa articulação para o planejamento de propostas de ensino-aprendizagem no serviço, através de métodos que proporcionem a produção do cuidado pelos residentes.

Durante o primeiro ano (R1), o residente de enfermagem participa de atividades teórico-práticas com temas transversais para todas as categorias profissionais, tais como: Ética, Bioética e Sociedade; Bioestatística e Epidemiologia; Metodologia Científica; Gestão nos Serviços de Saúde; SUS e Políticas Públicas de Saúde; Biossegurança nos serviços de saúde; Educação em Saúde; Seminário Temático; e na área de concentração, Política de Atenção à Saúde em Ortopedia e Traumatologia. 
Já o segundo ano (R2) é voltado para temas específicos destinados a atuação da enfermagem, como: Sistematização da Enfermagem na Rede de Atenção em Ortopedia e Traumatologia; Gestão da Enfermagem na Rede de Atenção em Ortopedia e Traumatologia; e Enfermagem na Promoção da Saúde em Ortopedia e Traumatologia. Para a conclusão da residência, de acordo com o regimento interno, deve ser produzido o Trabalho de Conclusão da Residência (TCR), uma monografia ou artigo científico, realizado a partir de orientação a pesquisa. É importante destacar que, devido a pandemia da Covid-19, a RMS teve que adaptar e modificar em alguns momentos o cronograma de prática, além disso, as atividades teórico-práticas foram realizadas principalmente em formato remoto online, mas a carga horária foi cumprida integralmente.

Assim, a atividade prática é desenvolvida por meio do treinamento em serviço através de rodízios, para que o residente vivencie a rotina e assista pacientes com afecções ortotraumatológicas em variadas unidades de atuação hospitalares: Ambulatório, Central de Material e Esterilização, Pronto Socorro, Centro Cirúrgico, Clínica Pediátrica, Médica e Clínica Cirúrgica. Vale ressaltar que, mesmo não estando no rodízio outros setores, como por exemplo, Unidade de Terapia Intensiva, quando tem-se fluxo de pacientes ortopédicos, o residente de enfermagem pode ser solicitado para a assistência hospitalar.

Porém, a atuação na atenção básica não está inclusa nos rodízios, sendo um desafio ao programa para que o residente tenha atuação profissional em todos os níveis de atenção do SUS, visto que propõem a formação para o atendimento integral ao paciente. Além disso, a RMS está em uma região que também atende populações com saberes e práticas tradicionais, pacientes que trazem aspectos peculiares da cultura amazônida, de grande importância na formação do residente (Pereira et al., 2018).

As aulas são desenvolvidas no decorrer da semana, dependendo da disponibilidade dos docentes, a partir de exposição, pesquisas de artigos, discussão de casos clínicos, entre outas metodologias, podendo algumas disciplinas serem ministradas no período da noite, após o serviço, assim, em alguns momentos pode-se ultrapassar 60 horas de carga horária semanal, tornando-se extenuante. Nesse sentido, Silva (2018) afirma que a carga horária de 60 horas semanais é incompatível com as propostas de formação para o trabalho em saúde, que de certa forma caracteriza-se como um instrumento de educação em serviço pautado na integralidade e interdisciplinaridade, e por outro lado cria uma carga horária exaustiva que gera precarização e exploração do trabalho. Assim, mesmo havendo particularidades na residência, com atividades práticas e teórico-práticas, estas não alteram sua condição de trabalhador.

Além disso, Cavalcanti e colaboradores (2018) abordam fatores que podem prejudicar o desempenho do residente no programa de RMS como, a falta de experiência profissional, carga horária semanal elevada e a jornada de estudante e trabalhador podem gerar Síndrome de Burnout, com sintomas como exaustão emocional, baixa realização do trabalho e depressão aos residentes das diferentes categorias profissionais.

\subsection{Atuação do enfermeiro residente em Ortopedia e Traumatologia no serviço e na atenção à saúde}

A atuação do enfermeiro residente em Ortopedia e Traumatologia no Oeste do Pará perpassa por diversos setores de atendimento ao paciente com afeç̧ões traumato-ortopédicos. No R1 a vivencia inicia-se na clínica médica e cirúrgica, com a prestação de cuidados diretos aos pacientes no pré e pós-operatório de cirurgias ortopédicas. Na clínica do HRBA o residente acompanha o paciente desde sua admissão, com a realização de consulta de enfermagem, exame físico, orientações quantos aos cuidados e rotina da instituição, até a sua alta hospitalar para seguimento ambulatorial, assim como também atua em questões gerenciais a partir de orientações do enfermeiro do setor, como a elaboração de escalas de serviço e solicitações de materiais para a assistência.

Já no HMS, é realizada a visita diária aos pacientes internados nas clínicas médica, cirúrgica e pediátrica, entre médicos, enfermeiros e fisioterapeutas, ambos residentes, a qual proporciona troca de experiências quanto aos casos clínicos e estado de saúde dos pacientes, mas ainda falta a participação de profissionais do hospital para uma maior integração ensino- 
serviço. Em algumas situações, mesmo o residente estando inserido em um hospital escola, há a necessidade de esclarecimento quanto aos objetivos da RMS, a formação e atuação profissional no serviço.

Nesse aspecto, Mello et al., (2018) afirmam que, para a integração ensino-serviço ocorrer, necessita-se de transformação nas práticas de ensino, cuidado e gestão na área da saúde, que poderiam facilitar a formação profissional do residente de acordo com os princípios e diretrizes do SUS. Assim, o aperfeiçoamento da inserção desses residentes nos cenários de prática contribuirá para a melhoria do cuidado multiprofissional, sendo que o atendimento de modo individualizado deve dar espaço a atuação conjunta para uma prestação de cuidados com qualidade a todos os pacientes ortopédicos na instituição (Nascimento et al., 2021).

No Centro Cirúrgico de ambos campos de prática durante a residência, a atuação do enfermeiro é realizada ao lado do profissional médico, especialistas e residentes em Ortopedia e Traumatologia. Em que o papel do residente de enfermagem é voltado ao preparo do paciente para a cirurgia, desde a degermação do membro a ser operado, participação em cirurgias ortopédicas como auxiliar e/ou instrumentador cirúrgico, realização de imobilização ortopédica quando necessária ao final do procedimento, e cuidados de recuperação pós-anestésica, possibilitando uma cirurgia segura e sem danos ao paciente. O residente interage com a equipe de saúde, principalmente com técnicos de enfermagem das salas operatórias, mas não tem um contato direto com o gerenciamento do cuidado quanto ao preenchimento de instrumentos de gestão.

Após a realização do procedimento cirúrgico, o paciente é direcionado para o seguimento ambulatorial com retornos agendados de um atendimento para outro, onde são assistidos para uma recuperação rápida e eficaz até sua alta. No ambulatório de ortopedia o residente de enfermagem pode realizar curativos, retiradas de pontos, imobilizações, além de orientações aos cuidados com o membro afetado no domicílio, para a prevenção de infecções no pós-operatório, uso de medicações e ao retorno para reavaliação. Nalin et al., (2014) aborda que é de grande importância a promoção do autocuidado por meio da motivação/responsabilização para a recuperação do seu estado de saúde, mesmo que o paciente tenha limitações.

No HMS também são prestados cuidados de enfermagem ao paciente na urgência/emergência com trauma ortopédico. Nesse setor há um fluxo muito grande de pacientes, principalmente politraumatizado, onde a atuação do residente envolve a avaliação inicial de enfermagem e confecção de imobilizações, quando necessário, de acordo com o membro lesionado e quadro clínico do paciente, pois deve-se levar em consideração alguns pontos, como por exemplo, as proeminências ósseas, lesões de pele e limitação na articulação. Assim, a imobilização geralmente é realizada com talas gessadas do tipo axilopalmar, antibraquiopalmar, tipo tubo, suropodálica, cruropodálica, velpeau, entre outras. Posteriormente, ao processo de imobilização, são realizadas objetivas orientações quanto aos cuidados, como, não molhar o gesso, elevar membro imobilizado e curativo diário se apresentar ferimento no local imobilizado.

Em cada setor específico há um preceptor, profissionais enfermeiros na assistência em saúde que ensinam e supervisionam o residente para a sua atuação profissional. Observa-se que em alguns locais de atuação, o preceptor é especialista em ortopedia e traumatologia e contribui fortemente na formação do residente, porém em outros há pouca interação entre preceptor no planejamento e no acompanhamento das atividades. Ressalta-se que os preceptores devem aguçar a reflexão dos profissionais em formação sobre suas práticas, visto que o ensino nos serviços de saúde gera um potencial questionador capaz de produzir conhecimento e garantia de uma assistência de qualidade e com base científica (Souza et al., 2019, Carneiro et al., 2021).

Assim, durante a residência, o enfermeiro realiza o manuseio de materiais utilizados na assistência aos pacientes, como gessos, fios, fixadores externos, caixas de fragmentos com placas, hastes e parafusos para osteossíntese, e de instrumentos específicos como, furadeiras, alicates e pinças, além de realizar variados procedimentos que não é possibilitado na graduação. Nesse sentido, Santos et al. (2021) afirmam que o paciente ortopédico necessita de cuidados específicos de 
enfermagem, visto que traz consigo um conjunto de aparatos, fixadores externos, aparelhos gessados, tração esquelética e pinos que podem ocasionar limitação de suas atividades diárias de autocuidado.

Portanto, observa-se que nas instituições de atuação da RMS há grande fluxo de pacientes com afecções ortopédicas e traumatológicas, sendo campos ricos em termos de conhecimentos e práticas de enfermagem. Nesse aspecto, o residente egresso tem um destaque em seu currículo, visto que possui formação de acordo com os princípios e diretrizes do SUS, com habilidades específicas, de acordo com as necessidades da região (Oliveira et al., 2017, Mello et al., 2021). Ademais, constatou-se a partir da vivência que a maior demanda de atendimento é composta por pacientes adultos jovens, do sexo masculino com histórico de acidente de motocicleta, com acometimento ortopédico principalmente relacionado a fraturas expostas e/ou fechada em membros inferiores. Dados que estão de acordo com publicações recentes sobre a temática (Abreu et al., 2019, Mendonça et al., 2021).

No contexto atual da pandemia COVID-19, durante a realização da residência foi vivenciado medo e incertezas sobre o futuro na formação, com uma série de transformações no cenário de atuação do residente como, implementação de novos fluxos de atendimento, avaliação de enfermagem aos pacientes ortopédicos sintomáticos e racionamento de equipamentos de proteção individual, além de desafios e dificuldades neste período de pandemia, como adoecimento físico e mental. Ribeiro et al. (2020) destaca que a pandemia afetou imensamente os serviços de saúde, necessitando de força de trabalho, estrutura física e insumos para o enfrentamento.

Por fim, o processo de avaliação da prática do residente é realizado ao término de cada rodízio pelo preceptor, no qual é estabelecida um conceito de 0 a 10 para cada critério: responsabilidade; criatividade; iniciativa; trabalho em equipe; capacidade de comunicação verbal e não verbal; e relações humanas. Também tem a participação do próprio residente, com a autoavaliação, o que possibilita uma reflexão.

\section{Considerações Finais}

Os resultados apresentados possibilitou a socialização de experiências vivenciadas fomentando a reflexão crítica da atuação do residente de enfermagem no programa de Residência Multiprofissional de Saúde em Atenção Integral em Ortopedia e Traumatologia, sendo uma oportunidade significativa de aquisição de conhecimentos técnicos-científicos que possibilita uma nova conduta diante da prática profissional, tornando o profissional apto a prestar uma assistência segura e sistematizada no âmbito do Sistema Único de Saúde.

Considerando o impacto das alterações musculoesqueléticas na saúde e a necessidade do aprimoramento do enfermeiro para assistir o paciente integralmente, o estudo oportunizou compreender a importância da atuação do residente de enfermagem no cuidado ao paciente com afecções ortotraumatológicas. Nesse sentido, a partir da experiência vivenciada foi possível acompanhar um elevado número de pacientes com diferentes lesões ocasionadas por variados fatores, mostrando que o residente contribui significativamente no serviço de saúde e atenção aos usuários, sendo a integração do ensino com o serviço uma estratégia de transformação da assistência à saúde e pacientes os principais beneficiados.

\section{Referências}

Abreu, M.S.S, Porto, T.N.R.S., Ferreira, M.T.A, \& Neves, N.V.P., Baldoino, L.S., Martins, V.S., Carvalho, D.P., Alcântara, S.M.L. (2019). Perfil das vítimas de acidente motociclístico atendidas pelo serviço de atendimento móvel de urgência. Revista Eletrônica Acervo Saúde, 37, e1560. https://doi.org/10.25248/reas.e1560.2019.

Arnemann, C.T., Kruse, M.H.L., Gastaldo, D., Jorge, A.C.R., Silva, A.L., Margarites, A.G.F., Pires, C.L., Kuplich, N.M., Santos, M.T., \& Condessa, R.L. (2018). Práticas exitosas dos preceptores de uma residência multiprofissional: interface com a interprofissionalidade. Interface (Botucatu), $22,1635-1646$. https://doi.org/10.1590/1807-57622017.0841. 
Arnemann, C.T., Kruse, M.H.L., da Silva, M.E.K., Terra, M.G., Mello, A. de L., da Silva, D.T., \& Souto, V.T. (2021). Sonhos de preceptores para um programa de residência multiprofissional: utopias a serem investidas? Pesquisa, Sociedade e Desenvolvimento, 10(7), e27010716079. https://doi.org/10.33448/rsd-v10i7.16079.

Barbosa, B. L. da S., Valentim, L.de A., Marinho, D. F., Martins, N. V. do N., Oliveira, S. M. S., Meschede, M. S. C., \& Costa, T.L.S. da (2021) Perspectivas e Desafios da Fisioterapia em um Programa de Residência Multiprofissional em Saúde em Santarém, Oeste do Pará, Amazônia. Pesquisa, Sociedade e Desenvolvimento, 10(12), e433101220709. https://doi.org/10.33448/rsd-v10i12.20709.

Brasil. (2006). Departamento de Gestão da Educação na Saúde. Residência multiprofissional em saúde: experiências, avanços e desafios. Ministério da Saúde. https://bvsms.saude.gov.br/bvs/publicacoes/residencia_multiprofissional.pdf.

Carneiro, E. M., Teixeira, L. M. S., \& Pedrosa, J. I. S. (2021). A Residência Multiprofissional em Saúde: expectativas de ingressantes e percepções de egressos. Physis, 31(3), e310314, https://doi.org/10.1590/S0103-73312021310314.

Cavalcanti, I. L., Lima, F. L. T., Souza, T. A, \& Silva, M. J. S. (2018) Burnout e depressão em residentes de um programa multiprofissional em oncologia: estudo longitudinal prospectivo. Revista Brasileira de Educação Médica, 42(1), 185-196. https://doi.org/10.1590/1981-52712018v42n1RB20170078

Conselho Federal de Enfermagem. Resolução no 422, de 04 de novembro de 2012. http://www.cofen.gov.br/resoluo-cofen-n-4222012_8955.html.

Dias, I. M. A. V., Pereira, A. K., Batista, S. H. S. S., \& Casanova, I. A. (2016). A tutoria no processo de ensino-aprendizagem no contexto da formação interprofissional em saúde. Saúde em Debate, 40(111), 257-67.

Galvão, C. P. (2017). A gestão do ensino na Residência Multiprofissional em Saúde no HRBA/Santarém: contribuições para a consolidação da cultura de hospital ensino na Amazônia (Dissertação de mestrado) - Pós-Graduação em Educação - Universidade Federal do Oeste do Pará, Santarém. http://www.ufopa.edu.br/ppge/images/dissertacoes/turma_2015/claudileia_pereira_galvao.pdf.

Mello, A. L., Terra, M. G., Nietsche, E. A., Siqueira, D. F., Canabarro, J. L., \& Arnemann, C. T. (2018). Formação de residentes multiprofissionais em saúde: limites e contribuições para a integração ensino-serviço. Revista de Enfermagem do Centro-Oeste Mineiro, 8, e2567. https://doi.org/10.19175/recom.v8i0.2567.

Mendonça, B. M. P., de Souza, N. K. T., Borges, J. H. S., \& Neto, J. S. de A. (2021). Perfil do condutor de moto vítima de acidente de trânsito no distrito federal. Brasília Med, 58, 1-6. https://doi.org/10.5935/2236-5117.2021v58a43.

Mussi, F. F., Flores, F. F., Almeida, C. B. (2021). Pressupostos para a elaboração de relato de experiência como conhecimento científico. Revista Práxis Educacional, 17(48), 60-77. https://doi.org/10.22481/praxisedu.v17i48.9010.

Nalin, V., Machado, J. B, Reichert, S. C., dos Santos, E. F., Ascari, R. A, Alves, J. P., Guralski, G., \& Oliveira, C. R. O. (2014). Atuação de enfermagem em serviço ambulatorial de traumato-ortopedia. Rev. Teor. Prát. Educ. 3(1), 05-11.

Nascimento, A. C. B.,do, \& Omena, K. V. M. (2021). A Educação Interprofissional em Programas de Residência Multiprofissional em Saúde no Brasil: Uma revisão integrativa. Research, Society and Development, 10, e8010413655, 10.33448/rsd-v10i4.13655. https://rsdjournal.org/index.php/rsd/article/view/13655.

Oliveira, J. B., Ceretta, L. B., Birolo, I. V. B., Simões, P. W., \& Sônego, F. G. F. (2017). Influência da residência multiprofissional na vida profissional de egressos. Revista Inova Saúde. Criciúma, 6(1), 122-139.

Ossege, C. L., Santos, O. M., Franco, A. C. D., Hoepfner, N. O., Carvalho, D. S. B., Moraes, G. H., Rego, E. R. M., Machado, A. C., \& Ronca, D. B. (2020). Atuação do profissional de saúde residente no enfrentamento da covid-19: um relato de experiência da Secretaria de Estado de Saúde do Distrito Federal. Cenas Educacionais, 3, e8489.

Pereira, M. N. S, Nascimento Junior, J. C. M., \& Galvão, E. F. C. (2018). A visão dos preceptores a respeito da residência multiprofissional com foco na ortopedia e traumatologia no interior da Amazônia. Rev. Exitus, 8(1). https://doi.org/10.24065/2237-9460.2018v8n1id400.

Ribeiro, A. P., Oliveira, G. L., Silva, L. S., \& Souza, E. R. (2020). Saúde e segurança de profissionais de saúde no atendimento a pacientes no contexto da pandemia de Covid-19: revisão de literatura. Rev Bras Saude Ocup, 45, e25. https://doi.org/10.1590/2317-6369000013920

Robleda, G., Sillero-Sillero, A., Puig, T., Gich, I., \& Baños, J. E. Influence of preoperative emotional state on postoperative pain following orthopedic and trauma surgery. (2014). Rev Latino-Am Enfermagem, 22(5), 785-91, 2014. https://doi.org/10.1590/0104-1169.0118.2481.

Santos, S. M. F., Lima, M. M. M. A., Santos, L. A., Silva, E. M. R., Carvalho Jr, A. M., \& Matos, R. R. C. (2021). Assistência de enfermagem a pacientes ortopédicos em um hospital público do oeste paraense. Pesquisa, Sociedade e Desenvolvimento, 10(17), e180101724536. https://doi.org/10.33448/rsdv10i17.24536.

Seltenreich, L. S. (2017). Competências do enfermeiro na prática da preceptoria na Residência Multiprofissional em Saúde (Dissertação de mestrado) - PósGraduação em Enfermagem - Faculdade de Medicina, Universidade Estadual Paulista "Júlio de Mesquita Filho", São Paulo. https://repositorio.unesp.br/handle/11449/150278.

Silva, L. B. (2018). Residência Multiprofissional em Saúde no Brasil: alguns aspectos da trajetória histórica. Revista Katálysis, 21(1), 200-209. https://doi.org/10.1590/1982-02592018v21n1p200.

Silva, L. S. \& Natal, S. (2019). Residência Multiprofissional em Saúde: análise da implantação de dois programas pela Universidade Federal de Santa Catarina, Brasil. Trab Educ Saúde, 17(3), e0022050.

Silva, P.C. (2017). Residência de enfermagem em ortopedia e traumatologia: experiência de implantação e desafios (Monografia de Enfermagem) Universidade Federal de Santa Catarina, Florianópolis. https://repositorio.ufsc.br/handle/123456789/173567. 
Research, Society and Development, v. 11, n. 3, e18411326547, 2022

(CC BY 4.0) | ISSN 2525-3409 | DOI: http://dx.doi.org/10.33448/rsd-v11i3.26547

Souza, S. V. \& Ferreira, B. J. (2019). Preceptoria: perspectivas e desafios na Residência Multiprofissional em Saúde. ABCS Health Sciences, 44(1), 15-21. https://dx.doi.org/10.7322/abcshs.v44i1.1074.

Torres, R.B.S., Barreto, I.C.H.C., Freitas, R.W.J.F., \& Evangelista, A.L.P. (2019). Estado da arte das residências integradas, multiprofissionais e em área profissional da Saúde. Interface, 23, e170691. 